\title{
Barriers to incident reporting in a healthcare system
}

\author{
R Lawton, D Parker
}

Qual Saf Health Care 2002;11:15-18

See end of article for authors' affiliations .....................

Correspondence to: Dr R Lawton, School of Psychology, University of Leeds, Leeds LS2 9JT, UK; rebeccal@

psychology.leeds.ac.uk

Accepted for publication 28 November 2001

\begin{abstract}
Background: Learning from mistakes is key to maintaining and improving the quality of care in the NHS. This study investigates the willingness of healthcare professionals to report the mistakes of others. Methods: The questionnaire used in this research included nine short scenarios describing either a violation of a protocol, compliance with a protocol, or improvisation (where no protocol exists). By developing different versions of the questionnaire, each scenario was presented with a good, poor, or bad outcome for the patient. The participants $(n=315)$ were doctors, nurses, and midwives from three English NHS trusts who volunteered to take part in the study and represented $53 \%$ of those originally contacted. Participants were asked to indicate how likely they were to report the incident described in each scenario to a senior member of staff.

Results: The findings of this study suggest that healthcare professionals, particularly doctors, are reluctant to report adverse events to a superior. The results show that healthcare professionals, as might be expected, are most likely to report an incident to a colleague when things go wrong $(F(2,520)=82.01$, $p<0.001)$. The reporting of incidents to a senior member of staff is also more likely, irrespective of outcome for the patient, when the incident involves the violation of a protocol $(F(2,520)=198.77$, $p<0.001$. It appears that, although the reporting of an incident to a senior member of staff is generally not very likely, particularly among doctors, it is most likely when the incident represents the violation of a protocol with a bad outcome.

Conclusions: An alternative means of organisational learning that relies on the identification of system (latent) failures before, rather than after, an adverse event is proposed.
\end{abstract}

A survey of the literature on human factors ${ }^{1}$ revealed that human error is the major contributory factor in about $80 \%$ of accidents in hazardous technologies. This claim, however, conveys little of the complexity of human failures. Many large organisations-for example, Shell Petroleum, British Rail, British Airways-have recognised the need to make the distinction between active and latent failures ${ }^{2}$ and, by doing so, have adopted a less punitive approach to error management. The distinction between active and latent failures acknowledges that it is not only those people at the sharp end of the system-pilots, operators, and doctors-who make errors, but that mistakes made by people remote from the workplace-such as managers, designers, and accountants-have a significant role in accident causation. These decisions can result in latent failures that are present within the system and can lead to local workplace factors that predispose individuals at the sharp end to unsafe acts (active failures). For example, the decision at a senior level not to replace a faulty piece of equipment might lead to the misreading of a dial (error) or the switching off of an alarm (violation). Latent failures, although critical in the accident causation system, are more difficult to identify than active failures whose consequences are usually immediate and evident. It is proposed that this systems approach to accident investigation, adopted in other industries, is equally applicable to analysing adverse events in clinical medicine. ${ }^{3}$

To achieve success through this approach, organisations must learn from their incidents, whatever form they take (adverse events, complaints, near misses). NHS reporting systems currently provide an incomplete picture of the scale and nature of failures in health care. In a recent document published by the Department of Health, ${ }^{4}$ plans for improvements to NHS reporting systems are outlined. As well as having in place effective information systems, learning from past failures also requires that the reporting of such events is reliable. However, there is evidence that underreporting is a significant problem, particularly for non-confidential report- ing systems. ${ }^{5-7}$ This underreporting is not only a problem for the NHS; Barach and Small reported that underreporting of adverse events in the USA is $50-96 \%$ annually. ${ }^{8}$

The culture of medicine-with its emphasis on professional autonomy, collegiality, and self-regulation' -is unlikely to foster the reporting of mistakes. Moreover, the organisational culture of the NHS, with its emphasis on blame, ${ }^{4}$ and an increasingly litigious public may only serve to exacerbate the problem. Research in the USA ${ }^{10}$ suggests that, although errors in medicine are common and can lead to significant patient injuries, there are legal impediments to adopting the kind of error reduction strategies that have proved successful in other industries. The reporting of errors is crucial to the process of error management, but physicians with tort liability concerns may be reluctant or unwilling to do so, given legal rules which grant the plaintiff's attorney access to this information. Together, the culture of the medical profession which discourages reporting and increasing fears of litigation are therefore likely to constrain the reporting of errors in the NHS.

Moreover, the increasing drive towards evidence-based medicine, clinical guidelines, and protocols means that medical incidents, when they occur, may be attributed to deviations from, or violations of, the best practice defined in writing. ${ }^{11}$ Although subject to the same influences as other errors such as poor decision making or planning, violations of best practice are often intentional and, as such, are considered more culpable. ${ }^{12}{ }^{13}$ It should be noted that, when used by the human factors community, the term "violation" does not necessarily imply law breaking but may merely reflect "deliberate (though not necessarily reprehensible) deviations from those practices believed necessary to maintain the safe operation of a potentially hazardous system" ${ }^{14}$

The study reported here investigates the willingness of healthcare professionals (doctors, nurses, and midwives) to report colleagues to a superior member of staff following an adverse incident or near miss. The study further explores the difference in reporting of events involving three kinds of 
Box 1 Example scenario

A nurse is setting up a drip for a female patient who needs a blood transfusion following an operation. The blood is the patient's own, taken before the operation commenced. The protocol states that the details of the blood should be checked by two staff members to minimise the risks of giving the patient the wrong blood. The nurse sees that her colleagues are all tied up with an emergency and therefore she checks the blood carefully herself and gives it to the patient. The patient is given blood of a different type by mistake and she needs emergency treatment.

behaviour distinguished by Reason et al ${ }^{15}$ — compliance with a protocol, violation of a protocol, and improvisation where no protocol exists.

\section{METHODS}

Participants in the study, carried out in early 1998, were 315 healthcare professionals of all grades in three specialties (surgery, anaesthetics, and obstetrics) from three English NHS trusts. Potential participants were initially contacted via hospital risk managers who supported the research. The purpose of the study was explained and those who were interested volunteered to complete the questionnaire. This yielded a total of 260 completed questionnaires. This number was increased by personal approaches from the researchers, particularly targeting staff in anaesthetics where numbers were low. In order to maintain confidentiality, non-responders (those who took a questionnaire but failed to complete and return it) were not followed up as they could not be individually identified.

Each questionnaire comprised nine short scenarios describing the behaviour of a nurse, doctor, or midwife in a particular situation such as the catheterisation of a spinal injury patient. The practice was depicted as either being in line with a protocol (compliance), a deliberate deviation from the protocol (violation), or as using clinical judgement where no protocol existed (improvisation). ${ }^{14}$ The example shown in box 1 describes the violation of a protocol in surgery with a bad outcome. Further example scenarios are shown in appendix 1. All the scenarios used in the study were developed in consultation with healthcare professionals who had taken part in an earlier phase of the research. ${ }^{13}$

Nine versions of the questionnaire were produced to allow for each scenario to be presented with a good, bad, and poor outcome for the patient. For the purposes of this survey the operational definition of a poor outcome included additional and avoidable discomfort for the patient, while a bad outcome included more prolonged and serious discomfort, short of fatality. The severity of the outcome, defined in terms of the actual effect on the patient rather than any potential effect, differed across scenarios. In one case a bad outcome involved emergency resuscitation, in another it involved having to return to hospital following discharge. The good, poor, and bad outcomes relating to each scenario were developed in consultation with healthcare professionals from the relevant specialties during a preliminary qualitative study. ${ }^{13}$

Having nine versions of the questionnaire also allowed us to control for the effects of order of presentation of the scenarios. For each respondent the nine scenarios in their version of the questionnaire covered all possible combinations of behaviour (violation, compliance, and improvisation) and outcome (good, bad, or poor). Thus, the design allowed for the investigation of some incidents that, though involving rule breaking or erroneous decisions, did not lead to poor outcomes. These can be conceptualised as near misses. ${ }^{8}$

Respondents were asked to read each scenario and imagine that they had witnessed the events described. They were then asked a series of questions including "If you saw this happening how likely is it that you would report what you had seen to a superior?" Responses were indicated on a 5 point scale with end points labelled "very unlikely" (1) to "very likely" (5). Other questions included ratings of the appropriateness of the behaviour described, risk associated with the behaviour and responsibility for the outcome. These issues have been addressed in an earlier paper comparing public and professional perceptions. ${ }^{16}$

\section{Data analysis}

The data were analysed using a repeated measures analysis of variance (ANOVA) with two within subject factors (behaviour and outcome, each with three levels) and one between subject factor (professional group, also with three levels (nurse, doctor, midwife)). Inspection of the mean scores by professional group allowed us to investigate the willingness of each group to report colleagues to a superior. Repeated measures ANOVA also enabled us to compare ratings of the three types of behaviour independent of outcome, of outcome independent of behaviour, and the interaction of behaviour, outcome, and professional group.

\section{RESULTS}

Three hundred and fifteen healthcare professionals completed and returned the questionnaire. The final sample (representing a 53\% response rate) included 73 doctors, 145 nurses, and 92 midwives.

A repeated measures ANOVA revealed that there was a significant main effect for outcome $(\mathrm{F}(2,520)=82.01, \mathrm{p}<0.001)$. Post hoc tests showed that reporting of the practice of another healthcare professional was significantly more likely if the scenario described a bad outcome than a poor outcome, which in turn was more likely to lead to a report than a good outcome. There was also a significant main effect for behaviour type $(\mathrm{F}(2,520)=198.77, \mathrm{p}<0.001)$ and post hoc tests indicated that the violation of a protocol was more likely to be reported than improvisation (the use of clinical judgement), which in turn was more likely to be reported than compliance. There were also significant differences between the different professional groups $(\mathrm{F}(2,260)=27.01$, $\mathrm{p}<0.001)$ with doctors generally being less likely to make a report than either nurses or midwives. Table 1 shows the mean likelihood ratings for reporting the observed behaviour to a superior by professional group, behaviour type, and outcome.

Table 2 shows that, when the scenarios described either compliance with a protocol or improvisation in situations where no rule exists, healthcare professionals were unlikely to indicate that they would report what they had witnessed to a senior colleague. This was the case even when the outcome in

Table 1 Mean (SE) likelihood of reporting by professional group, behaviour type, and outcome

\begin{tabular}{llll}
\hline \multirow{2}{*}{$\begin{array}{l}\text { Respondent } \\
\text { type }\end{array}$} & \multicolumn{3}{l}{ Behaviour type } \\
\cline { 2 - 4 } & Compliance & Improvisation & Violation \\
\hline $\begin{array}{l}\text { Doctor } \\
\text { Good }\end{array}$ & $1.37(0.15)$ & $1.64(0.17)$ & $2.10(0.19)$ \\
$\begin{array}{l}\text { Poor } \\
\text { Bad }\end{array}$ & $1.71(0.20)$ & $1.95(0.19)$ & $2.56(0.19)$ \\
Midwife & $2.05(0.20)$ & $2.12(0.19)$ & $2.97(0.18)$ \\
Good & $1.24(0.13)$ & $1.96(0.15)$ & $3.29(0.17)$ \\
Poor & $2.00(0.17)$ & $2.40(0.17)$ & $3.57(0.17)$ \\
Bad & $2.43(0.18)$ & $3.01(0.17)$ & $3.85(0.16)$ \\
Nurse & $1.85(0.10)$ & $1.83(0.11)$ & $3.15(0.13)$ \\
Good & $2.41(0.13)$ & $2.54(0.13)$ & $3.74(0.13)$ \\
Poor & $2.57(0.14)$ & $3.20(0.13)$ & $4.15(0.12)$ \\
Bad & & & \\
\hline & & &
\end{tabular}


Table 2 Mean (SE) likelihood of reporting by professional group and behaviour type

\begin{tabular}{llll}
\hline \multirow{2}{*}{$\begin{array}{l}\text { Respondent } \\
\text { type }\end{array}$} & \multicolumn{3}{l}{ Behaviour type } \\
\cline { 2 - 4 } & Compliance & Improvisation & Violation \\
\hline Nurse & $2.28(0.08)$ & $2.53(0.08)$ & $3.68(0.08)$ \\
Midwife & $1.89(0.10)$ & $2.46(0.11)$ & $3.57(0.11)$ \\
Doctor & $1.71(0.11)$ & $1.90(0.12)$ & $2.54(0.12)$ \\
\hline
\end{tabular}

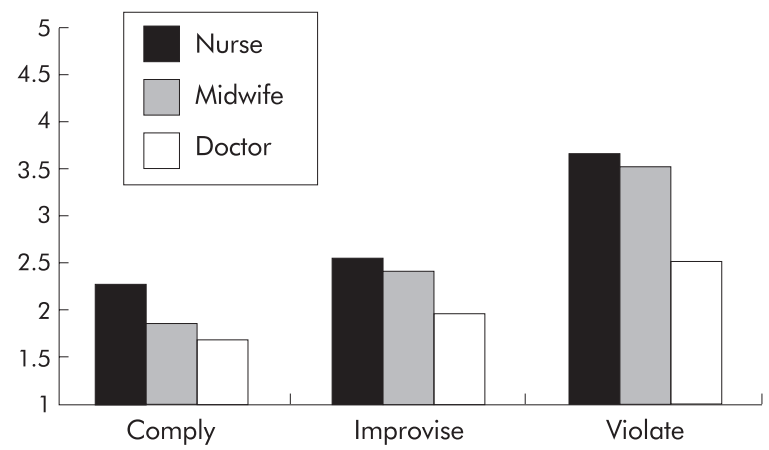

Figure 1 Likelihood of reporting compliance, improvisations, and violations by professional group.

the scenario was described as poor or bad. Doctors were unlikely to report what they had seen even when the behaviour described was a violation of a clinical protocol. This contrasts with the nurses and midwives who, on witnessing a violation, were significantly more likely to report what they had seen than were doctors. The same significant difference emerged for improvisations except that, in this case, no professional group was likely to make a report.*

Figure 1 shows the significant interaction between professional group and behaviour type $(\mathrm{F}(4,520)=6.48, \mathrm{p}<0.001)$. None of the other two or three way interactions were significant at $\mathrm{p}<0.05$.

\section{DISCUSSION}

This study has shown that both the type of behaviour/practice and its consequences for the patient are important influences on the likelihood that the behaviour of fellow healthcare workers will be reported. Moreover, the likelihood of reporting varied by professional group, suggesting that doctors, nurses, and midwives use different standards when making judgements about the behaviour of colleagues.

The results indicate that, when best practice is defined in the form of a written protocol, deviations from these are more likely to be reported, at least by nurses and midwives. This may be because professionals are unwilling to challenge the behaviour of a fellow professional without strong grounds and that protocols and guidelines, if they define best practice, provide such grounds. This is supported by the finding from an earlier focus group study that nurses use clinical protocols to support their arguments when challenging the practice of doctors. $^{13}$

*The sample of doctors included 25 surgeons, 33 anaesthetists, and 15 obstetricians. The repeated measures ANOVA was computed again to test for differences in reporting across these different specialties. There was no main effect for specialty $(F(2,56)=2.57, p=0.086)$. However, the pattern of means suggested that the biggest differences were for reporting of violations from protocols. In this context, those doctors working in obstetrics were most likely to indicate that they would report such an incident.
The results also suggest that healthcare professionals are, in general, reluctant to report behaviour that has negative consequences for the patient when that behaviour reflects either compliance with a protocol or improvisation where no protocol is in place. If substantiated, this would have implications for the management of safety in the NHS as, without informal reports of situations where compliance with a protocol has led to a bad outcome for the patient, efforts to improve existing protocols may be hampered. Improvisations with poor outcomes are also unlikely to be reported, which means that the organisation will be unable to learn from experience. Violations of clinical protocols are more likely to be reported, perhaps because the individuals concerned are perceived by colleagues to be more culpable. However, there are interprofessional differences. Even when the behaviour concerned reflects the deliberate violation of a clinical protocol, doctors are less likely than nurses or midwives to report colleagues to a superior. Their reluctance to report violations, even when the outcome for the patient is bad (mean $=2.97$ ) may be a function of the widespread and well documented resistance among doctors to clinical protocols which are perceived by many in the medical community as a threat to their professional autonomy. ${ }^{17}{ }^{18}$ Alternatively, the reluctance of doctors informally to report a colleague to a superior member of staff may simply reflect a professional culture in which what may be seen as whistle blowing is taboo.'

This study has some important limitations. It was not possible to draw a truly random sample and it is likely that those who responded were individuals with a particular interest in risk. It was also not possible to monitor differential response rates across the professional groups sampled. However, there was a reasonable number of responses from each of the three groups, providing sufficient power for comparisons across the groups. The use of scenarios can be criticised on the grounds that they lack realism. In order to minimise this possibility, the scenarios used were developed in collaboration with healthcare professionals and were based on situations that had actually been encountered. Moreover, the reliance on self-report always carries with it the possibility of social desirability effects. However, it is difficult to envisage an alternative methodology that would have been feasible in this context.

The study was conducted before the development of a national reporting system for adverse incidents in the NHS. It therefore asked about the likelihood of making a report to a superior member of staff, as case review and other forms of internal monitoring of adverse events frequently rely on this kind of reporting. However, further research is needed to investigate whether the findings here can be generalised to other forms of reporting such as confidential reporting.

Notwithstanding these limitations, the findings of this study warrant consideration. They suggest that some cultural change may be necessary in the NHS before either the informal reporting studied here or a more formal incident reporting system can work optimally. Work in other high risk organisations has shown that error management can be both proactive and reactive. ${ }^{2}$ If adverse incidents are to be minimised, the organisation must learn from its mistakes and this is the message of the recent DOH publication "An organisation with a memory". ${ }^{4}$ In the context of the NHS, it may be that a proactive error management system such as those used in other high risk industries ${ }^{2}$ that are designed to measure and reduce the adverse impact of latent failures within an organisation may be the answer. Such systems involve not only the reporting of negative consequences, but also learning from occasions when inappropriate behaviour/practice has gone unpunished-that is, more by luck than good judgement. Proactive systems work in part by asking people to judge how frequently each of a number of factors such as staffing, supervision, procedures, and communication impact adversely on a specific aspect of their work. So, for example, if nurses in intensive care are experiencing problems with the design of a 


\section{Key messages}

- Healthcare professionals appear reluctant to report adverse incidents to a senior member of staff.

- Doctors are more unwilling than nurses or midwives to report adverse incidents to a senior staff member.

- Reporting is most likely when the incident involves the deviation from a protocol and when the outcome for the patient is bad.

- An unwillingness to report incidents must be addressed if organisational learning is to be achieved in the NHS.

particular piece of equipment, this will be recorded and action taken to improve the design. This kind of proactive approach allows the identification of latent failures before they give rise to errors that compromise patient safety. Such a system may serve, not only to reduce error, but also to foster a culture that, by moving away from blaming the individual, encourages reporting. Although no such system currently exists in the NHS, much can be learnt from other industries that have already embraced this approach.

\section{APPENDIX 1 \\ EXAMPLE SCENARIOS}

\section{Obstetrics, improvisation with a good outcome}

A woman has been in the second stage of labour for over an hour. The midwife, who is concerned about the baby, calls on a doctor to assist in the delivery. There is no protocol to guide the doctor about the best method of assisting the delivery. The doctor decides to use forceps because he thinks that it is going to be necessary to rotate the baby's head. This is indeed the case and after only a few more minutes the baby is delivered.

\section{Surgery, compliance with a poor outcome}

A female patient requires an intravenous drug. Although the nurse is capable of doing this herself, the protocol states that a doctor must give the first dose of any drug administered intravenously. The nurse follows the protocol and sets up all the equipment and then bleeps a doctor. It takes a long time for a doctor to arrive. Eventually a doctor rushes onto the ward, sites the cannula, administers the drug, and rushes out again. The delay in siting the cannula and administering the drug means that the patient is more uncomfortable than she would have been.

\section{Anaesthetics, violation with a bad outcome}

A female patient is going down to theatre for breast surgery. The anaesthetist who is to perform the anaesthetic has just started for the day. The protocol states that the anaesthetist must check all the equipment that is used in the operating theatre at the beginning of each shift. The anaesthetist relies on the checks that the operating department assistant has made and does not perform any additional checks. During the operation the anaesthetist has to perform manual heart massage when both the ventilator and ventilator alarm fail.

\section{Authors' affiliations}

R Lawton, School of Psychology, University of Leeds, Leeds LS2 9JT, UK

D Parker, Department of Psychology, University of Manchester,

Manchester M13 9PL, UK

This work was funded by the Economic and Social Council (ESRC).

\section{REFERENCES}

1 Hollnagel E. Human reliability analysis: context and control. London: Academic Press, 1993

2 Reason J. Managing the risks of organisational accidents. Aldershot, UK: Ashgate: 1997.

3 Vincent C, Taylor-Adams S, Stanhope N. Framework for analysing risk and safety in clinical medicine. BM 1998;316:1154-7.

4 Department of Health. An organisation with a memory. London: The Stationery Office, 2000

5 Stanhope N Crowley-Murphy M, Vincent $C$, et al. An evaluation of adverse incident reporting. J Eval Clin Pract 1999;5:5-12.

6 Walker SB, Lowe M. Nurses' views on reporting medication incidents. Int J Nursing Pract 1998;4:97-102.

7 Weingart SN, Ship AN, Aronson MD. Confidential clinician reported surveillance of adverse events among medical inpatients. J Gen Intern Med 2000:15:470-7.

8 Barach P, Small SD. Reporting and preventing medical mishaps: lessons from non-medical near miss reporting systems. BN 2000;320:759-63.

9 Rosenthal M. How doctors think about medical mishaps. In: Rosenthal M, Mulcahy L, Lloyd-Bostock S, eds. Medical mishaps. Buckingham: Open University Press, 1999: 141-53.

10 Liang BA. Error in medicine: legal impediments to US reform. J Health Politics Policy 1999;24:27-58.

11 Hurwitz B. Guidelines and jurisprudence: advice, guidance or regulation. Epidemiol Preview 1998;22:85-93.

12 Lawton R. Not working to rule: understanding procedural violations at work. Safety Sci 1998;28:77-95

13 Lawton R, Parker D. Procedures and the professional: the case of the British NHS. Soc Sci Med 1999:48:353-61.

14 Reason JT, Manstead A, Stradling S, et al. Errors and violations on the roads: a real distinction? Ergonomics 1990;33:1315-32.

15 Reason JT, Parker D, Lawton R. Organisational control and the varieties of rule related behaviour. J Organisat Occup Psychol 1998;71:289304

16 Lawton R, Parker D. Judgements of the rule-related behaviour of healthcare professionals: an experimental study. $\mathrm{Br} J$ Health Psychol 2002 (in press).

17 Grol R. National standard setting for quality of care in general practice: attitudes of general practitioners and response to a set of standards. $\mathrm{Br} J$ Gen Pract 1990:40:361-4.

18 Tunis SR, Hayward RSA, Wilson MC, et al. Internists' attitudes about clinical practice guidelines. Ann Intern Med 1994;120:956-63. 